\title{
Incorrect Mathematical Conjecture
}

\section{Arij Zulfi Mufassaroh \& Adib Rifqi Setiawan}

A direct computer search yielded:

$$
55^{5}+3183^{5}+28969^{5}+85282^{5}=85359^{5}
$$

as the counterexample to a conjecture proposed by by Leonhard Euler in 1679 that at least $n n$th powers are required to sum to an $n$th power, $n>2$ (Singh, 2017, p. 213).

\section{Reference}

Singh, S. (2017). Fermat's Enigma: The Epic Quest to Solve the World's Greatest Mathematical Problem. Knopf Doubleday Publishing Group. 